\title{
DETERMINATION OF SENSOR DEPTH FROM DOWNWELLING IRRADIANCE MEASUREMENTS
}

\section{Philipp Groetsch}

\author{
Water Insight \\ Marijkeweg 22 \\ 6709PG Wageningen, The Netherlands \\ VU University Amsterdam \\ Institute for Environmental Studies (IVM) \\ De Boelelaan 1087 \\ 1081HV Amsterdam, The Netherlands
}

\author{
Peter Gege \\ German Aerospace Center (DLR) \\ Remote Sensing Technology Institute \\ 82234 Oberpfaffenhofen, Germany
}

\begin{abstract}
The wave focusing effect causes measurements of under water downwelling irradiances $E_{d}$ to be extremely variable in intensity and spectral shape. A new analytical model for $E_{d}$ has been developed recently, which can deal with the induced variability by treating the direct and diffuse components of $E_{d}$ separately. This paper presents a method to determine the current sensor depth from under water downwelling irradiance measurements using that model combined with improved parameterizations for the direct and diffuse downwelling attenuation coefficients. The average relative deviation of sensor depth for a data set containing over 1400 measurements under various illumination conditions and depths is on the order of $5 \%$.
\end{abstract}

Index Terms - Downwelling Irradiance, Wave Focusing, Sensor Depth, WASI

\section{INTRODUCTION}

In situ measurements are essential for the validation of water remote sensing algorithms. For shallow waters, the bottom reflectance has a significant influence to the remotely sensed signal. To calculate bottom reflectances accurately, the downwelling irradiance $E_{d}$ has to be measured under water. Under water measurements of $E_{d}$ can also be used to determine water constituent concentrations [1]. However, because of the wave focusing effect, measurements of $E_{d}$ are extremely variable [2]. A new analytical model by Gege [3] can correct for the wave focusing effect by treating the direct and diffuse components of $E_{d}$ separately. To improve the characterization of the attenuation for the direct and diffuse components of $E_{d}$ within that model, parameterizations

Thanks to the Limnological Station (Technical University Munich) in Iffeldorf for the pleasant cooperation. for the direct $\left(K_{d d}\right)$ and diffuse $\left(K_{d s}\right)$ fractions of $K_{d}$ have been developed by Groetsch [4]. By fitting the model parameters to under water downwelling irradiance spectra, an accurate estimation of the current sensor depth can be retrieved. Typically, $E_{d}$ sensors designed for under water measurements have a pressure sensor included to estimate the current sensor depth. However, those measurements usually have a resolution on the order of tens of centimeters, which can be critical for shallow water applications. Furthermore, the depth estimation from pressure sensors does not account for waves in the field of view of the $E_{d}$ sensor, which can change the optical path length considerably.

The method presented in this paper solely relies on the spectral information recorded in under water $E_{d}$ measurements and therefore offers redundancy to potentially existing depth information retrieved from pressure sensors. In this paper a data set, covering a variety of measurement conditions and sensor depths, is used to validate the developed models and to show the applicability of a sensor depth retrieval from under water $E_{d}$ measurements.

\section{MATERIALS AND METHODS}

\subsection{Measurements}

For the validation of the $E_{d}$ model by Gege [3] and the $K_{d d}$ and $K_{d s}$ parameterizations by Groetsch [4], a dataset of $E_{d}$ field measurements was collected with a Trios $^{1}$ RAMSES ACC VIS (wavelength range 320 - $900 \mathrm{~nm}$, spectral sampling interval $3.3 \mathrm{~nm}, \mathrm{SN}$ : 806f) spectroradiometer. Additionally, measurements of the upwelling radiance and irradiance, $L_{u}$ and $E_{u}$ were performed, but not used for this publication. The spectral calibration of the instrument was carried out by the

\footnotetext{
${ }^{1}$ http://www.trios.de
} 
sensor manufacturer (Carl Zeiss MicroImaging GmbH, Jena, Germany). The radiometric calibration was performed by Trios with a NIST-traceable FEL lamp (DXW-1000W, 120V) which is calibrated by Gigahertz-Optik GmbH (Türkenfeld, Germany) according to NIST standards. Because the radiometric calibration of the used irradiance sensor (RAMSES ACC-VIS, serial number: 806f) was carried out more than two years before the field measurements, the calibration was validated with a NIST-traceable FEL lamp (GAMMA SCIENTIFIC 5000-16C, serial number: GS1033, calibrated 16. Oct 2009). In the range of $400-800 \mathrm{~nm}$ used for this study, the spectral differences were below $4 \%$, which is considered negligible. As the absolute intensities are of no importance for the fit of sensor depth (section 2.2, [2]), the spectral and radiometric calibration by Trios was used for the data evaluation.

The measurements were performed in Lake Starnberg, which is located south of Munich, Germany. With an area of about $60 \mathrm{~km}^{2}$ and a north-south extent of more than 20 $\mathrm{km}$ it is the fifth biggest lake in Germany. The measurements were taken at two sites, a jetty in Starnberg ( $\mathrm{N} 47.9963^{\circ}, \mathrm{E}$ $11.3495^{\circ}$ : 'Mole Starnberg') at the northern shore and a jetty in Seeshaupt (N 47.8214 ${ }^{\circ}$, E 11.3208 ${ }^{\circ}$ : 'Lido Seeshaupt') at the southern shore. The setup in figure 1, consisting of a cantilever arm build onto a mobile construction, allowed for the successive submersion of the sensor mount into the water column. The mount is balanced out in the water, so that the sensors are aligned normally to the water surface. The entry optics of the up- and downwards measuring sensors are aligned in one horizontal plane. A measuring tape connects the cantilever arm with the sensor mount to have reliable, independent readings of the actual sensor depth. A source of error is the determination of the offset between the entry optics of the $E_{d}$ sensor and the the measuring tape, which is assumed to be on the order of $1 \mathrm{~cm}$. Currents may tilt the sensor mount and bulge the measuring tape. However, only little influence from currents was observed during the measurements and the corresponding uncertainty is estimated to be on the order of $1 \mathrm{~cm}$.

The profile measurement starts with the sensors above the water surface to capture the incident radiation $\left(E_{d}\left(0^{+}\right)\right)$ followed by a measurement just below the surface $\left(E_{d}\left(0^{-}\right)\right)$. Consequently, measurements in fixed depth intervals are taken until the sensor mount touches the ground. The last measurement is a second take of $E_{d}\left(0^{+}\right)$to check if the illumination conditions remained stable during the acquisition. Each measurement consists of at least 30 individual data takes to capture the present variability due to the wave focusing effect. In table 1 the details of the measurements are listed. The listed wave heights were taken as the uncertainty in the reading of the sensor depth from the measuring tape. The data set comprises a large variety of illumination conditions, concerning sun zenith angle and cloud cover. As the measurements were carried out from a jetty close to the shore, the maximal sampling depth was restrained by the local bathymetry to $2.77 \mathrm{~m}$.

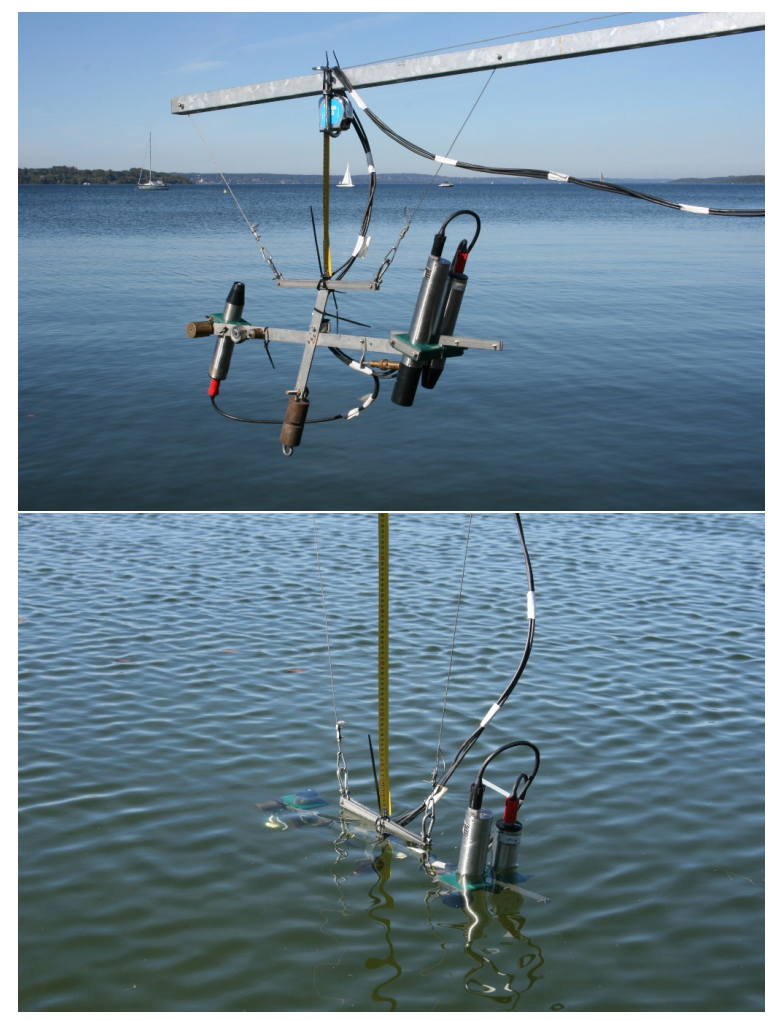

Fig. 1. Measurement setup: sensor mount (top), sensor mount partially submersed (bottom)

\subsection{Processing}

The new analytical $E_{d}$ model by [3] and the parameterization for $K_{d d}$ and $K_{d s}$ by [4] are implemented to the public domain software WASI [5]. Using WASI, the developed models were fitted to the measured $E_{d}$ spectra. The sensor depth is one of the parameters determined during those fits. The validation of the models was then carried out with the independent field measurements of sensor depth. The model used for the calculation of the downwelling irradiance just above the water surface is the analytic model of Gregg and Carder [6]. It assumes a cloudless, marine standard atmosphere. To achieve an optimal fit of the sensor depth, the atmospheric parameters in the Gregg and Carder model have to be adjusted to the the above water spectra. Those parameters are: ozone scale height $H_{o z}$, Angström exponent $\alpha$, turbidity coefficient $\beta$ and water vapor concentration WV. The determined values are averaged over all above water spectra and set constant for the subsequent under water fits. These were performed with the following parameters and start values:

- Concentration of phytoplankton class no. 4 (dinoflagellates) $C[4]=2.0 \mathrm{mg} \mathrm{m}^{-3}$ 
Table 1. Details of measurements at location 'Mole Starnberg' (M) and 'Lido Seeshaupt' (L)

\begin{tabular}{l|l|l|l|l|l} 
Parameter & M1 & M2 & M3 & L1 & L2 \\
\hline \hline Date, [dd-mm-yy] & $11-10-10$ & $16-06-11$ & $28-06-11$ & $04-10-10$ & $17-05-11$ \\
Local Time, [hh:mm] & $14: 15-15: 45$ & $14: 00-15: 30$ & $19: 45-21: 15$ & $15: 30-16: 30$ & $11: 30-13: 30$ \\
Depths, [cm] & $0-277$ & $0-243$ & $0-193$ & $0-131$ & $0-117$ \\
Depth steps, $[\mathrm{cm}]$ & 30 & 30 & 30 & 30 & 50 \\
Measurements, [\#] & 360 & 276 & 329 & 230 & 251 \\
Cloud cover, $\left.[\%]^{[}\right]^{\circ}$ & 0 & 100 & 0 & 0 & 100 \\
Sun zenith angle, $\left[{ }^{\circ}\right]$ & $57-65$ & $27-37$ & $78-90$ & $61-68$ & $29-34$ \\
Wave height, $[\mathrm{cm}]$ & 5 & 3 & 3 & 5 & 10 \\
Bottom type & macroph. & sediment & macroph. & macroph. & sediment
\end{tabular}

- Suspended matter concentration $C_{X}=0.6 \mathrm{~g} \mathrm{~m}^{-3}$

- Gelbstoff concentration $C_{Y}=0.3 \mathrm{~m}^{-1}$

- Spectral slope of Gelbstoff absorption $S=0.016 \mathrm{~nm}^{-1}$

- Depth $z=2.0 \mathrm{~m}$

- Fraction of direct radiation $f_{d d^{2}}{ }^{2}=1.0$

- Fraction of diffuse radiation $f_{d s}=1.0$

The sun zenith angle for each measurement was calculated automatically, based on location and local time. The values for the Q-factor and the water temperature were set to $Q=5$ and $\mathrm{T}_{w}=20.0^{\circ} \mathrm{C}$. The fits were performed in a wavelength range of 400 to $800 \mathrm{~nm}$ and an interval of $3 \mathrm{~nm}$. The quantity minimized during the fit procedure was set to least squares. The fit of the sensor depth is slightly dependent on the yellow substance exponent $S$. As independent measurements of $S$ were not performed within this study, $S$ was included as a fit parameter.

\section{RESULTS}

Figure 2 shows the results of a linear regression analysis of the independently measured sensor depths and the WASIdetermined depths. Each point in the graph corresponds to the mean over several data takes (usually 30 ) at a distinct depth. The errorbars correspond to the standard deviation of those data takes. The linear regression results in a $\mathrm{R}^{2}$ of 0.99 with a slope of 0.98 and an intercept of $6.49 \mathrm{~cm}(\mathrm{p}<0.01$, standard error $=0.02 \mathrm{~cm}$ ). In table 2 the absolute and relative standard deviations as well as the absolute and relative deviations from the measuring tape readings are listed. The same analysis has been performed with the widely used $K_{d}$ model by Gordon [7], which resulted in an average relative standard deviation of $30 \%$ and an average relative deviation of $29 \%$. The atmospheric parameters, determined by fitting the atmospheric model in WASI to the above water measurements, are listed in table 3 .

\footnotetext{
2 as explained in [3]
}

Table 2. Comparison of the WASI fit results with the measuring tape readings.

\begin{tabular}{l|rrrr} 
Site & $\begin{array}{r}\text { abs. STD } \\
{[\mathrm{cm}]}\end{array}$ & $\begin{array}{r}\text { rel. STD } \\
{[\%]}\end{array}$ & $\begin{array}{r}\text { abs. dev. } \\
{[\mathrm{cm}]}\end{array}$ & $\begin{array}{r}\text { rel. dev. } \\
{[\%]}\end{array}$ \\
\hline \hline L1 & 6.58 & 8.65 & 5.23 & 5.44 \\
L2 & 2.62 & 3.51 & 4.53 & 1.42 \\
M1 & 5.95 & 6.41 & -1.96 & -2.58 \\
M2 & 5.51 & 3.44 & 0.15 & -0.46 \\
M3 & 4.14 & 4.45 & 17.68 & 16.31 \\
\hline Abs. mean & 4.96 & 5.29 & 5.91 & 5.24
\end{tabular}

Table 3. Atmospheric parameters derived with WASI from above water $E_{d}$ measurements.

\begin{tabular}{l|rrrrr} 
& M1 & M2 & M3 & L1 & L2 \\
\hline \hline $\mathrm{H}_{\mathrm{oz}},[\mathrm{cm}]$ & 0.276 & 0.662 & 0.337 & 0.225 & 0.535 \\
$\alpha,[-]$ & -0.170 & 0.070 & -0.207 & 0.522 & 0.038 \\
$\beta,[-]$ & 0.455 & 1.598 & 0.461 & 0.226 & 1.837 \\
$W V,[\mathrm{~cm}]$ & 0.157 & 1.977 & 0.804 & 0.659 & 1.103
\end{tabular}

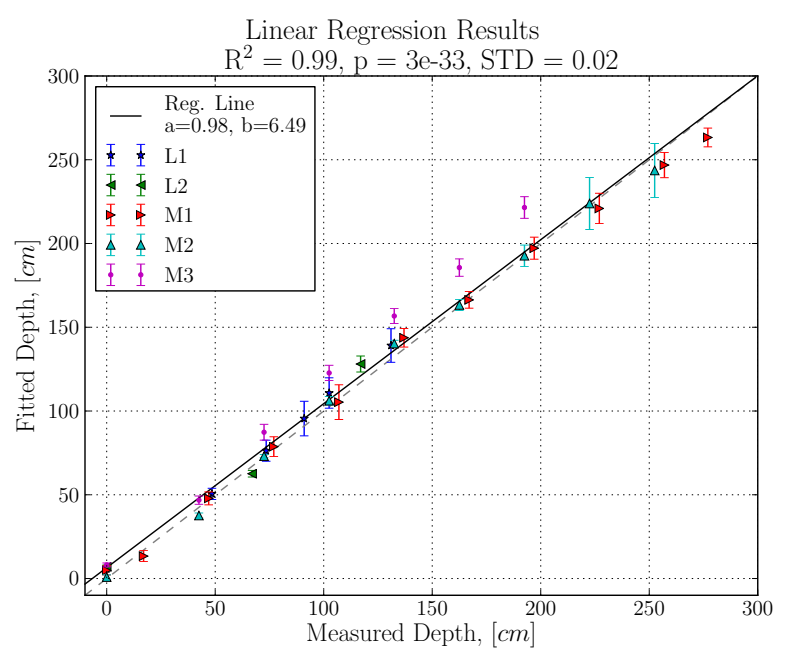

Fig. 2. Validation of WASI fits: Fit results for sensor depth plotted against measured sensor depth. 


\section{DISCUSSION}

The low average relative standard deviation on the order of 5 $\%$ indicates that variabilities in $E_{d}$, introduced by the wave focusing effect, are correctly interpreted by the model. The low average absolute deviations, on the order of $5 \mathrm{~cm}$, suggest that the method is suitable for the determination of the sensor depth from $E_{d}$ spectra. Even for the high sun zenith angle case $\mathrm{M} 3$, reasonable results are obtained. Considering the wave heights of $3-10 \mathrm{~cm}$ for the collected data set, an error of the same order of magnitude is expected for the independent readings of sensor depth from the measuring tape. As this error is of the same order of magnitude as the observed deviations, it can be regarded as an upper limit for the real uncertainty of the presented method. This conclusion is supported by the results of the linear regression analysis with a squared coefficient of correlation of 0.99 , a slope close to one and an interception on the order of the average wave heights. Those results make the method suitable for sensor depth dependent applications in shallow as well as in optically deep waters. It has to be stressed that a reliable sensor depth estimation cannot be achieved with conventional $K_{d}$ models, such as the widely used Gordon model, considering the resulting high standard deviations and deviations (both on the order of $30 \%$ in the analysis of the current data set).

As all the measurements were taken in the same lake, the optical properties of the sampled water columns are similar. Therefore a repetition of the presented validation measurements in highly turbid as well as extremely clear waters would be of interest. The maximal sampling depth of $2.77 \mathrm{~m}$ can be considered as sufficient for the investigated water bodies. However, for clear waters considerably higher sampling depths would be necessary to validate the model. An interesting consecutive application is the determination of inertial optical properties (IOPs) from $E_{d}$ spectra and even more, their change with depth.

\section{CONCLUSION}

The presented method for the determination of sensor depth from downwelling irradiance measurements has been proven to produce accurate results for various illumination conditions and sensor depths for the investigated water bodies. In contrast to sensor depths acquired from pressure sensors, the method does not require additional instrumentation and is sensitive to the actual optical path length as wave heights are taken into account. Those accurate measurements of sensor depth are important for the determination of water constituent concentrations and inherent optical properties from $E_{d}$ spectra and indispensable for calculating bottom reflectance, which is a key quantity in shallow water remote sensing applications.

\section{REFERENCES}

[1] P. Gege, "Estimation of phytoplankton concentration from downwelling irradiance measurements in water," The Israel Journal of Plant Sciences, in press.

[2] P. Gege and N. Pinnel, "Sources of variance of downwelling irradiance in water," Applied Optics, vol. 50, no. 15, pp. 2192-2203, 2011.

[3] P. Gege, "Analytic model for the direct and diffuse components of downwelling spectral irradiance in water.," Applied optics, vol. 51, no. 9, pp. 1407-1419, 2012.

[4] P. Groetsch, Optimization and Verification of a new Analytical Radiative Transfer Model, Diploma thesis, http://elib.dlr.de/71048/, 2011.

[5] P. Gege, "The water color simulator WASI: an integrating software tool for analysis and simulation of optical in situ spectra," Computers \& Geosciences, vol. 30, no. 5, pp. 523-532, 2004.

[6] W. Gregg and K.L. Carder, "A simple spectral solar irradiance model for cloudless maritime atmospheres," Limonology And Oceanography, vol. 35, no. 8, pp. 16571675, 1990.

[7] H.R. Gordon, "Can the Lambert-Beer law be applied to the diffuse attenuation coefficient of ocean water?," Limnology and Oceanography, vol. 34, no. 8, pp. 1389-1409, 1989. 